\title{
Binocnlares Cornealmikroskop.
}

\author{
Von \\ Dr. S. Czapski \\ in. Jena.
}

Mit 1 Figur im Text.

Die Bedeutung und der Werth des binocularen (plastischen) Sehens auch auf dem Gebiet der wissenschaftlichen mikroskopischen Forschung wird allmählich in immer weiteren Kreisen anerkannt. Die diesem Zweck dienenden, neuerdings von der Firma Carl Zeiss in den Handel gebrachten Instrumente (binoculare Präparirmikroskope verschiedener mechanischer Ausstattung ${ }^{1}$ )), haben sich geradezu überraschend schnell eingebürgert und die Vortheile, die ihre Anwendung für mancherlei $Z$ wecke gewährt, sind von den zuständigsten Beurtheilern wiederholt hervorgehoben bezw. anerkannt worden.

Für die Beobachtung der Cornea des lebenden Auges ist schon seit längerer Zeit ein binoculares Instrument in der Zehender-Westien'schen Loupe in Gebrauch und in der That lassen gerade bei diesem Object die Verhältnisse ein binoculares plastisehes Sehen ganz besonders angezeigt erscheinen, da die Durchsichtigkeit der Cornea ein genaues Localisiren von darin befindlichen Fremdkörpern oder normalen Gebilden für das monoculare Sehen sehr erschwert. Der Zehender-Westien'schen Loupe haften aber

1) S. Zeitschr. f. wissenschaftl. Mikroskopie. 14. 1897. S. 289 -313 und Katalog von Carl Zeiss über Mikroskope etc., 31. Ausg. 1898. S. $94-99$. 
mehrere Mängel an: einmal ist ihre Vergrösserung sehr gering, geht über 3-5 kaum himaus, ferner kann diese Vergrösserung nicht geändert, dem speciellen $Z$ weck angepasst werden, und endlich muss für die Beleuchtung der anvisirten Stelle immer erst besonders (durch Aufstellung von einer Art Belenchtungsapparat, in Lampe und Sammellinse bestehend) gesorgt werden. Der letztere Umstand macht den Apparat nuerwünscht sperrig und schwerfällig im Gebrauch.

Als daher vor einigen Jahren Herr Dr. Barkan, Professor an der Universität in San Francisco Cal. gelegentlich der Vorführung des vom Verfasser mit Dr. Schanz zusammen construiten monocularen Cornealmikroskops ${ }^{1}$ ), das Bedürfniss nach einem analogen binocularen Instrument betonte, hat die Firma Carl Zeiss gern dieser Anregung Folge gegeben. Die optische Seite der Aufgabe war durch die damals im wesentlichen schon vollendete Construction der stereaskopischen Präparirmikroskope nach Greenough und Braus-Drüner, bereits nahezu erledigt, and es bedurfte nur einer zweckmässigen, d. h. dem hier vorliegenden Gebrauchszweck angepassten Gestaltung des mechauischen Theils des Instruments, des Gestells.

Die nach einigen Erprobungen endgiltig angenommene Ausfïhrungsform ${ }^{2}$ ) des Instruments, ist in der Figur dargestellt. Das eigentliche Mikroskop, d. h. der die optischen Systeme enthaltende Theil desselben, ist im wesentlichen demjenigen der oben genannten gleich, die für andere wissen-

1) Vergl, die diesbezügl. Publieationen von Dr. F. Schanz in. Zehender's klin. Monatsbl. f. Augenheilk. März 1893. Verhandl. d. Gesellsch. deutscher Naturf. u. Aerzte. 1895. II. 2. Hälfte. S.194-196, sowie Knapp u. Schweigger's Arch. f. Augenheilk. Bd. 31. 1895.

2) Ein sich von diesem endgiltigen nicht viel unterscheidendes Exemplar wurde von Herrn Dr. Schanz der in August 1898 in Heidelberg tagenden Versammlung deutscher Augenarzte vorgeführt, s. den Bericht über dieselbe. 
schaftliche und technische Zwecke dienen. Eine nähere Darlegung der für die Construction dieses optischen Theils maassgebenden Gesichtspunkte ist vom Verfasser in der Zeitschrift f., wissenschaftl. Mikroskopie 1. c. gegeben, worauf

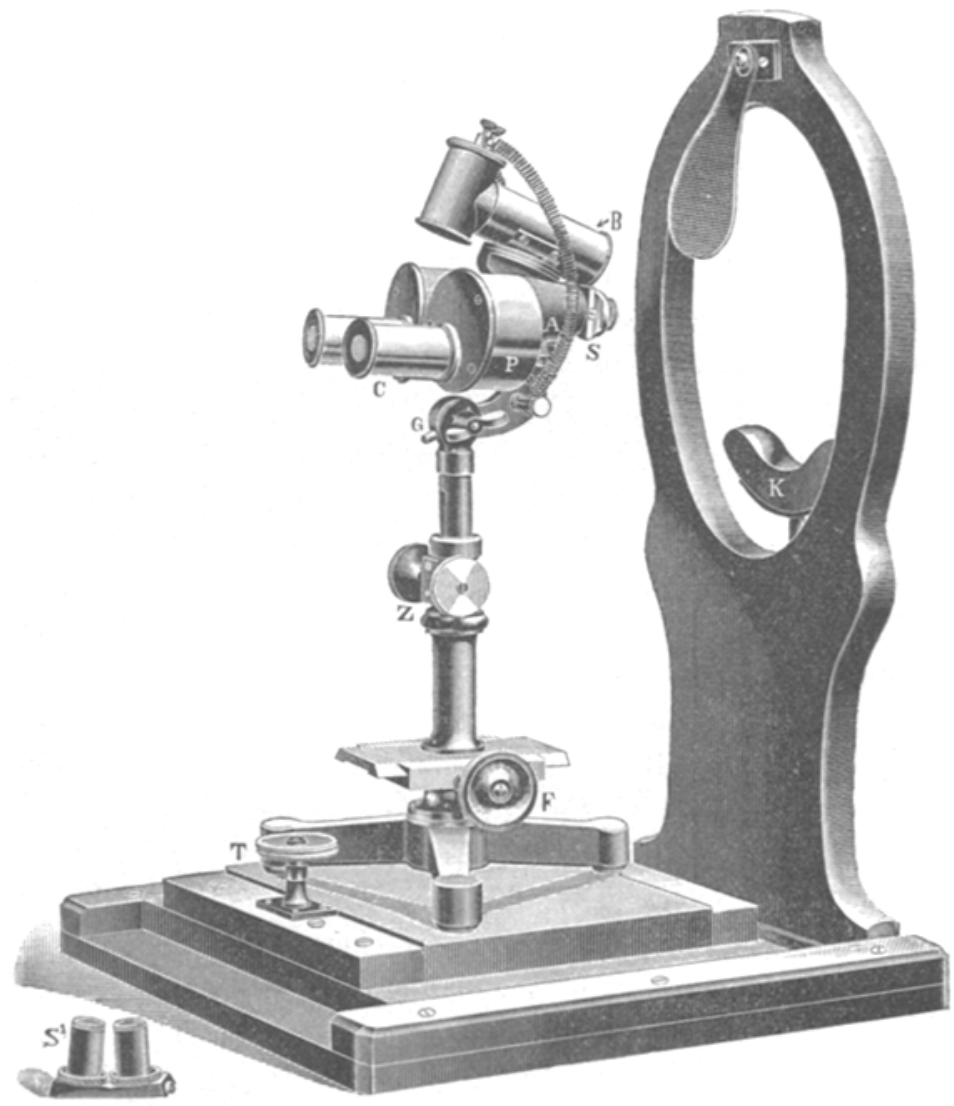

hier verwiesen werden muss. Das Instrument besteht aus zwei vollständigen, unter einem Winkel von etwa $16^{\circ} \mathrm{zu}$ einander geneigten Mikroskoprohren, die in ihrem unteren, objectseitigen, Theil in einem (Aluminium-) Gusskörper $A$ verbunden sind. Der mittlere Theil wird durch je eine 
cylindrische Buichse $P$ gebildet, in der sich ein bildaufrichtendes Prismensystem nach Porro befindet. Die Oculare $C$ sind (in federnden Hülsen) ebenso wie die objectseitigen Rohrenden $A$ excentrisch an den Bïchsen $P$ angebracht, wie dies durch die Lage der in letzteren befindlichen Prismen und den Strahlengang in diesen bedingt wird. Dadurch ist aber zugleich ein bequemes Mittel für die Anpassung des Ocularabstandes an den Augenabstand des Beobachters gegeben, indem die Oculare sammt den Biichsen $P$ rm die Ansatzstellen von $A$ freihändig drehbar eingerichtet werden.

Der Hauptvortheil der gewählten Construction besteht nun eben in der Anwendung je eines vollständigen Mikroskops für jedes Auge behufs Erzielung des binocularen (plastischen) Sehens statt der zu diesem Zweck früher fast ausschliesslich angewandten Theilung der von nur einem $\mathrm{Ob}$ jectiv aufgenommenen Strahlenbuischel und Ueberführung dieser (mittelst Spiegel, Prismen u. dergl.) in getrennte Ocularrohre. Gegenüber dieser Einrichtung ist die gewählte in allen maassgebenden Beziehungen erheblich im Vortheil, wie hier nicht näher ausgeführt werden soll. Insbesondere ist die Bildqualitä bei ihr eine bessere und der stereoskopische Effect vollkommener. Durch Anwendung der bildaufrichtenden Prismen (in den Büchsen P) ist daneben erreicht, dass an diesen Mikroskopen die Vor:züge des zu wissenschaftlichen $Z$ wecken so gut wie ausschliesslich angewandten sogenannten zusammengesetzten Mikroskops - insbesondere dessen grosses Sehfeld - zugleich mit der für alles Manipuliren so werthvollen Eigenschaft der aufrechten Abbildung zur Geltung kommen.

Am anderen Ende von $A$ finden die Objective Platz und zwar neuerdings in der Art, dass sie paarweise in ihrer genau richtigen gegenseitigen Stellung auf je einen kleinen Schlitten $S$ montirt sind; dieser lässt sich in ein an $A$ befestigtes Mutterschlittenstick bis zu einem festen An- 
schlag hineinschieben und durch Hinausschieben leicht wieder daraus entfernen, - ganz ähnlich wie bei der Schlittenwechselvorrichtung, mit der die Firma Carl Zeiss seit länger als einero Jahrzehnt ihre monocularen Mikroskope besonders zum Mikrophotographiren ausstattet. Es könmen so auf's Bequemste Objectivpaare verschiedener Stärke, die in gleicher Art auf Schlitten montirt sind, angewandt 1) und durch deren Wechsel die Vergrösserung variirt werden. Tm etwaige Verschiedenheiten im Refractionszustande der Augen des Beobachters zu. compensiren, kann für jedes Auge einzeln scharf eingestellt werden. $Z$ u diesem $Z$ weeke ist das rechte Objectiv jedes Paares fur sich von einer Mittellage aus heraus- und hereinschraubbar. Man verfăhrt nun beim erstmaligen Gebrauch eines Objectivpaars in der Art, dass man auf ein gut definirtes Object, etwa ein Stück gedrucktes - vorübergehend an der Augenklappe des Kinnhalters befestigtes Papier - erst durch Bewegen des ganzen Mikroskops mittels des Triebknopfes $T$ für das linke Auge scharf einstellt, und dann für das rechte Auge durch Herein- oder Herausschrauben des rechten Objectivs bei ungeänderter Lage des ganzen Mikroskopes. Durch freihändiges Aufeinanderzu - oder Voneinanderwegbewegen der beiden Oculare stellt man sodann den dem Augenabstand entsprechenden Ocularabstand her. Die richtige Regulirung dieser Verbältnisse giebt sich dem Beobachter sogleich durch den markanten stereoskopischen Effect zu erkennen, der auch bei einem beliebigen Stück Papier an dessen Fasern, Lettereindrücken u. s. w. zu erkennen ist. Ein zweites Mittel zur Veränderung der Vergrösserung ist der Wechsel der Oculare, welche letztere natürlich eben-

1) In der Figur ist ein solches Objectivpaar, das schwächste, am Mikroskop in der Gebrauchslage dargestellt, ein anderes stärkeres $S^{\prime}$ neben demselben liegend. Ueber die Construction der Objective berichtet $H$. Harting in Zeitschrift für wissenschaft1. Mikroskopie. 15. 1898. S. $299-303$. 
falls dem vorliegenden Zweck besonders angepasst sein müssen, damit die Qualität des stereoskopischen Bildes keine Einbusse erfährt. Die auf diese Weise erreichbaren auf den Normalabstand von $25 \mathrm{~cm}$ bezogenen Vergrösserungen und zugehörigen freien Objectabstände giebt die nachstehende Tabelle an.

Tabelle der freien Abstände und Vergrösserungen.

\begin{tabular}{c|c|c|c}
\hline \multirow{2}{*}{$\begin{array}{c}\text { Objectiv- } \\
\text { bremnweite }\end{array}$} & \multicolumn{2}{|c|}{ Vergrösserung wit: } & $\begin{array}{c}\text { Arbeitsabstand : } \\
\text { (zwischen anvisirtem } \\
\text { Auge und Objectiv) }\end{array}$ \\
\hline \hline $55 \mathrm{~mm}$ & Ocular z & Ocular 4 & $75 \mathrm{~mm}$ \\
45 & 9 & 16 & 56 \\
35 & 26 & 28 & 43 \\
30 & 33 & 41 & 28
\end{tabular}

Es braucht wohl kaum besonders hervorgehoben zu

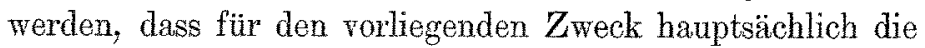
schwächeren von diesen Vergrösserungen praktisch in Betracht kommen. Doch erlauben die relativ grossen, freien Abstände, darin ziemlich weit zu gehen.

Oberhalb des Doppelrohres $A$, in der Mittelebene desselben, ist das Beleuchtungsrohr $B$ angeordnet, das nach demselben Punkt (dem Mittelpunkt des Objectfeldes) gerichtet ist, wie die beiden Mikroskoprohre. Es trägt am einen Ende eine kleine Glihlampe, im Innern einige Belenchtungslinsen, die das Licht der ersteren in angemessener Concentration auf die anvisirte, d. h. die Kreuzungsstelle der beiden Mikroskopazen werfen. So ist also, wie bei dem friher von mir mit Dr. Schanz zusammen construirten monocularen Cornealmikroskop stets, wie man das Mikroskop auch drehen und wenden mag, die anvisirte Stelle des Objects, wenn sie scharf erscheint, auch in der besten Beleuchtung. Es sind während der Beobachtung keinerlei Manipulationen bierfür nöthig, und die Aufmerksamkeit des Arztes kann sich ungetheilt dem Patienten zuwenden. 
Das ganze Mikroskop, Tuben sammt Beleuchtungsrohr, ist in nahezu ausbalancirter Stellung in der Verticalebene um ein festklemmbares Gelenk $G$ mit horizontaler Axe drehbar, desgleichen um eine verticale Axe. Es kann ferner mittels der Zahn- und Triebvorrichtung $Z$ in der Höhenrichtung, mittels des Zahn- und Triebs $F$ von vorn nach hinten verstellt werden, so dass die interessirende Stelle mit aller Sicherheit und Bequemlichkeit eingestellt werden kann. Der ganze anf einem massiven Dreifuss sich erhebende Apparat wird zweckmässig auf dem Grundbrett eines Kinnhalters nach Art der von Jung-Heidelberg construirten aufgestellt. Der in der Figur dargestellte unterscheidet sich von diesem hauptsächlich durch das Grundbrett, das auf Vorschlag von Prof. Eversbusch in zwei zu einander senkrechten Richtungen beweglich eingerichtet ist. Die Bewegung des Brettes und damit des Instruments von vorn nach hinten geschieht freihändig, durch einfaches Verschieben, die von rechts nach links, mittels des Triebknopfs $T$. 